Review

\title{
Mechanism of vascular endothelial dysfunction causing atherosclerosis: vascular endothelial glycocalyx
}

\section{Running title: vascular endothelial glycocalyx damage in atherosclerosis}

\author{
Minako Yamaoka-Tojo, MD and $\mathrm{PhD}^{1,2}$
}

1 Department of Rehabilitation/Regenerative Medicine and Cell Design Research Facility, Kitasato University School of Allied Health Sciences, Sagamihara, Japan

2 Department of Cardiovascular Medicine, Kitasato University Graduate School of Medical Sciences, Sagamihara, Japan

* Corresponding author: Minako Yamaoka-Tojo, MD, PhD; E-mail: myamaoka@med.kitasato-u.ac.jp; Phone: (81)-42-778-8111, Fax: (81)-42-778-9696;

\begin{abstract}
In atherosclerosis patients, vascular endothelial dysfunction is commonly observed with damage of vascular endothelial glycocalyx, an extracellular matrix-bound to and encapsulating the endothelial cell lining the blood vessel wall. Unfavorable lifestyle; smoking and physical inactivity, also induces glycocalyx degradation. Moreover, the vascular endothelial glycocalyx is damaged by various unfavorable disease conditions like as dehydration, acute infectious disease, trauma, sepsis, ARDS, Kawasaki disease, preeclampsia, gestational diabetes mellitus, hypertension, diabetes, chronic kidney disease, atherosclerosis, stroke, dementia, microvascular angina, acute coronary syndrome, and heart failure. The vascular endothelial glycocalyx has been shown to be important not only as a physical cytoprotective barrier for vascular endothelial cells but also as a mechanism that regulates intracellular cell signaling. Therefore, vascular endothelial glycocalyx has great potential to explore new strategies for assessing the benefit conditions of our healthy vasculature.
\end{abstract}

Keywords: vascular endothelial function; inflammation; oxidative stress; cardiovascular disease prevention; disease management

\section{Vascular endothelial cell dysfunction}

The blood vessels that extend all over the body consists of one layer of vascular endothelial cells. Vascular endothelial cells are known to produce various physiologically active substances and are responsible for homeostasis in the living body. If one person's blood vessels are connected together, the length will be two and a half rounds of the earth, and if all the vascular endothelial cells forming the inside of the blood vessel are put together into one, it is comparable to the largest organ which is larger than the liver.

Vascular endothelial cells have various functions such as inflammation/anti-inflammation, coagulation/fibrinolysis system, promotion/inhibition of angiogenesis, etc. By regulating these balance, vascular endothelial cells are responsible for maintaining homeostasis in the body (Fig. 1). Healthy vascular endothelial cells exhibit a good vascular endothelial function. Coronary risk factors like as smoking, hypertension, diabetes, dyslipidemia, obesity, and physical inactivity are major factors that cause vascular endothelial dysfunction and further promote atherosclerosis progression (Fig. 2). The 
comprehensive disease management, such as smoking cessation and improvement of lifestyle-related diseases, makes it possible to maintain a good vascular endothelial function.

Figure 1. Vascular endothelial dysfunction.Vascular endothelial dysfunction is equal to vascular endothelial cell dysfunction which leads to vascular failure, which cannot keep the balance of good vascular conditions.

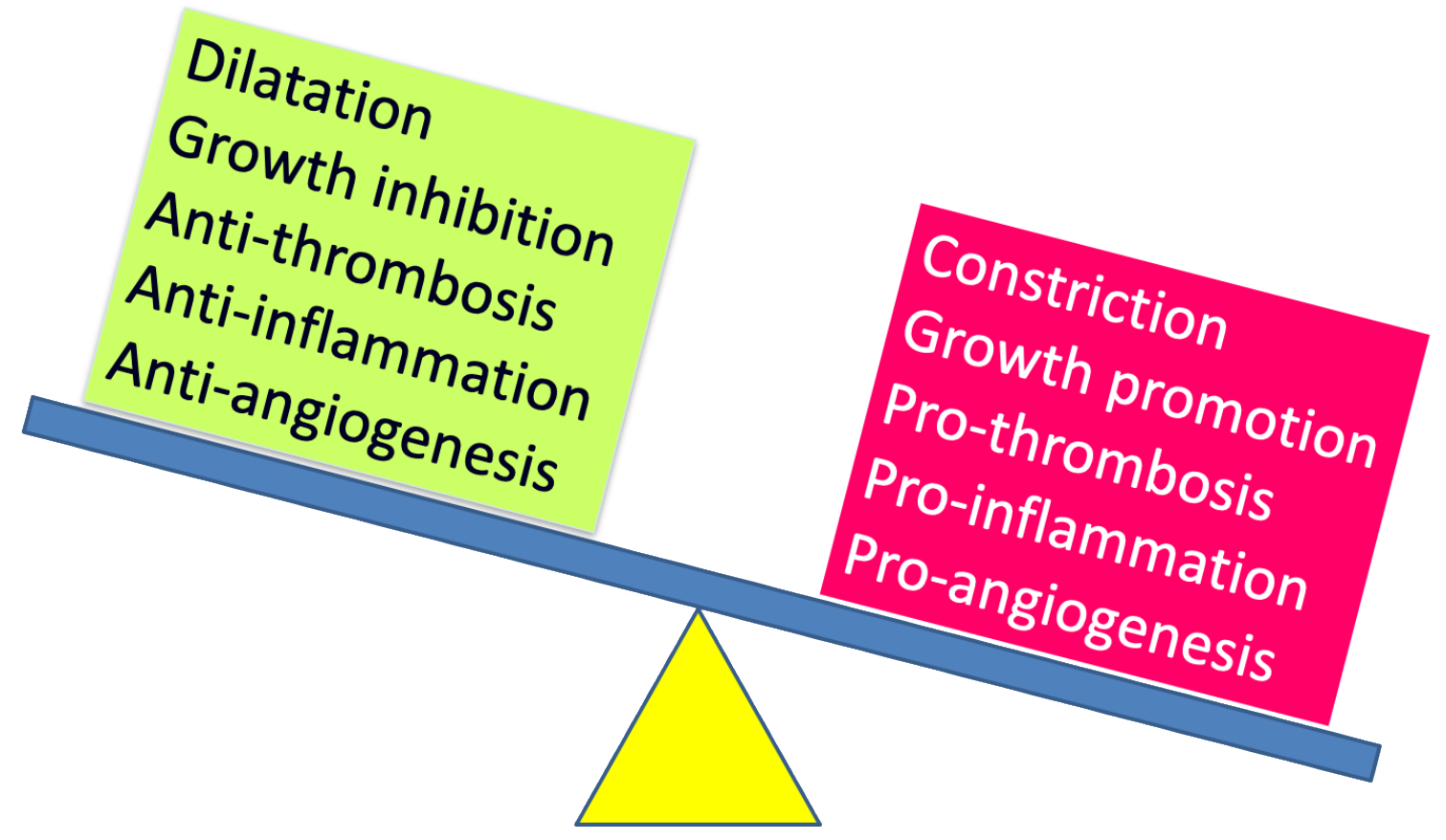

Figure 2. Endothelial dysfunction induces atherosclerosis progression. The vascular endothelial function might have potential prognostic value for the early detection of atherosclerosis. FFA, fatty free acids; ROS, reactive oxygen species, Ox-LDL, oxidized-low density lipoprotein. 


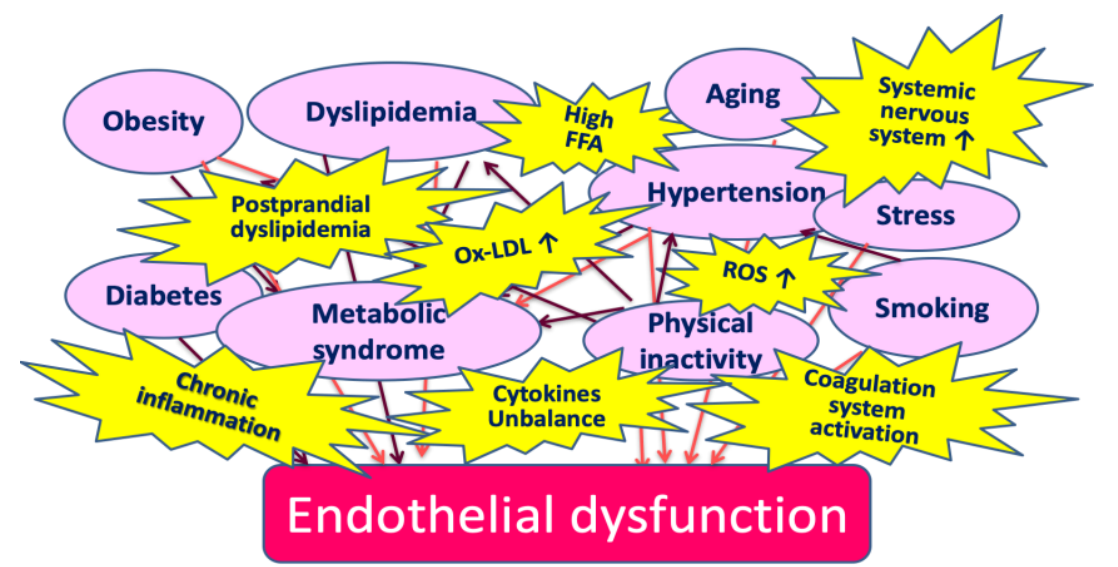

$\square$

Atherosclerosis 
Vascular endothelial function test has been used as an indicator to predict the onset of future cardiovascular events and determine the effectiveness of comprehensive disease management in cardiovascular medicine. Although it has said that "humans grow older with blood vessels", the vascular endothelial function is an integrated index showing the health of blood vessels, and maintaining a good vascular endothelial function leads to the health of the living body. To elucidate these precise mechanisms, we should research more about the molecular mechanisms of vascular endothelial cell function.

\section{Endothelial glycocalyx and atherosclerosis}

The barrier of extracellular polymeric compounds consists of glycoproteins that form a 200-500 nm glycosylated gel layer that covers vascular endothelial cells. The gel layer is called vascular endothelial glycocalyx. In a healthy vascular endothelial cell covering with enough amount of glycocalyx, cells are well organized with a good vascular endothelial function such as producing nitric oxide (NO), anticoagulant factors, and a sufficient amount of reactive oxygen species (ROS) to maintain vascular homeostasis (Fig. 3A).

Figure 3. Vascular endothelial glycocalyx covering vascular endothelial cells. A. A healthy vascular endothelial cell covering with enough amount of glycocalyx. Endothelial cells covered with glycocalyx are well maintained in endothelial function producing nitric oxide (NO), anticoagulant factors, and a sufficient amount of reactive oxygen species (ROS) to maintain vascular homeostasis. B. An unhealthy vascular endothelial cell partly covered thinned glycocalyx. In vascular endothelial cells in which vascular endothelial glycocalyx is damaged by hyperglycemia or oxidized LDL, excessive ROS are produced, and NO production is reduced, which promotes inflammatory cytokines releasing and coagulation. LOX-1, lectin-like oxidized LDL receptor; VCAM-1, vascular cell adhesion molecule-1; PAI1, plasminogen activator inhibitor-1; TF, tissue factor; vWF, von Willebrand factor; ox-LDL, oxidized low-density lipoprotein; eNOS, endothelial NO synthase, MMPs, matrix metalloproteases; tPA, tissue plasminogen activator; PGI2, prostacyclin; TM, thrombomodulin.

A.

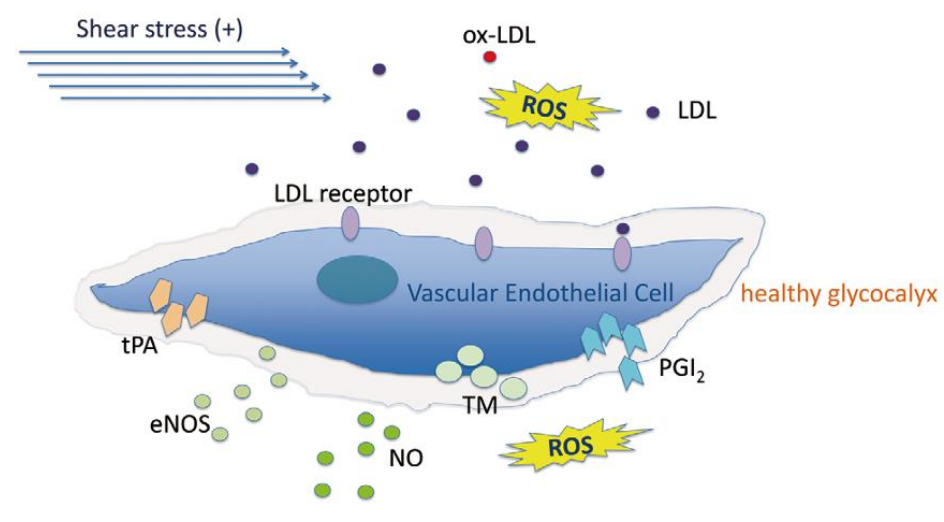

B.

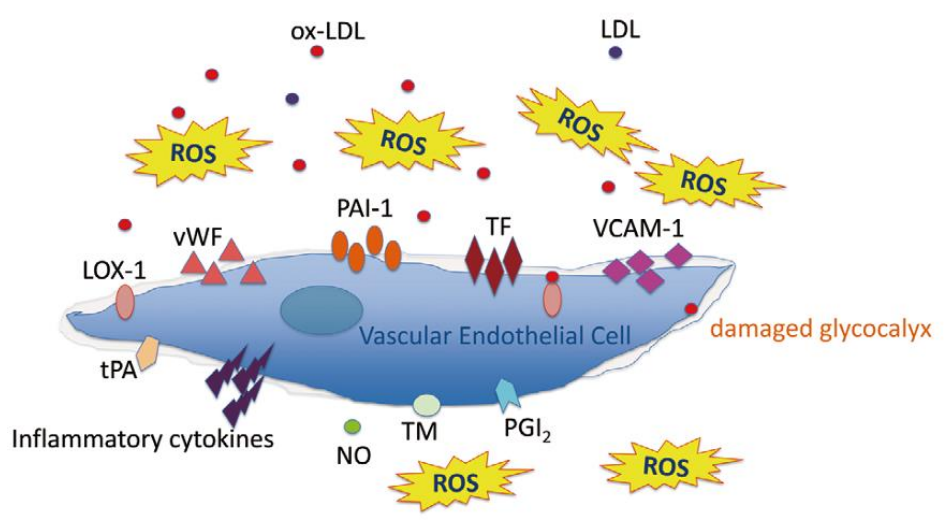


The glycocalyx is composed of proteoglycans and glycosaminoglycans. In the group of proteoglycans, there are syndecans such as syndecan-1 (CD138) and syndecan-4, glypican, mimecan, perlecan, and biglycan. In the glycosaminoglycans, hyaluronan (including hyaluronic acid and hyaluronate), heparin, heparan sulfate, chondroitin, dermantan sulfate, keratan sulfate, are included. The vascular endothelial glycocalyx plays important roles to cover physically vascular endothelial cells, maintain cell-cell adhesion of vascular endothelial cells, and control the cell signaling among vascular endothelial cells, which may affect cancer metastasis, angiogenesis, and vascular permeability. The vascular endothelial glycocalyx is necessary to maintain the function of vascular endothelial cells which is also necessary to keep homeostasis in the body ${ }^{1}$.

The vascular endothelial glycocalyx is involved in the control of intracellular signal transduction such as proliferation/differentiation/apoptosis of vascular endothelial cells, cytoskeleton, thrombus formation, inflammatory/anti-inflammatory control, and immunity. Attention has been focused on the relationship with the pathogenesis of arteriosclerosis, and it has been suggested that it is associated with the progression of atherosclerotic diseases such as plaque formation and progression, thrombus formation, plaque rupture, and the pathogenesis of cardiovascular events. The vascular endothelial glycocalyx is impaired by endotoxin, inflammation, hypernatremia ${ }^{2}$, oxidized-LDL 3 , hyperglycemia, excessive fluid infusion, dehydration, and reduction of vascular wall shear stress. These stimulations systemically induce shedding of vascular endothelial glycocalyx and the glycocalyx layer is thinned under these conditions (Fig. 3B). In vascular endothelial cells partly covered by damaged endothelial glycocalyx, inflammatory cytokines are released a lot, excessive ROS are produced, and NO production is reduced, which promotes atherosclerosis progression. Importantly, vascular endothelial glycocalyx damage has already been reported in patients with diabetes ${ }^{4,5}$, inflammatory disease ${ }^{6}$, Kawasaki disease $^{7}$, sepsis ${ }^{8}$, ischemia/reperfusion injury ${ }^{9}$, trauma ${ }^{10}$, acute lung injury (ALI)/acute respiratory distress syndrome (ARDS) ${ }^{11}$, preeclampsia ${ }^{12}$, familial hypercholesterolemia ${ }^{13}$, chronic heart failure ${ }^{14}$, microvascular angina ${ }^{15}$, and cardiogenic shock ${ }^{16}$. It has been suggested that vascular endothelial glycocalyx damage is involved in the onset/deterioration mechanism of these diseases. In these patients, the fragmented vascular endothelial glycocalyx is detected at high concentrations in their blood and urine, and their vascular endothelial glycocalyx layer is thinned. In addition, coarse and fragile glycocalyx layer-induced by vascular endothelial glycocalyx damage is more frequently observed in these patients. Furthermore, by improving the pathological condition through therapeutic intervention, etc., these vascular endothelial glycocalyx damage can be ameliorated. Therefore, as a target for preventive measures for the onset and exacerbation of these diseases and new therapeutic strategies, vascular endothelial glycocalyx research is going on progressing.

The vascular endothelial glycocalyx is observed to be impaired from the early stage of arteriosclerosis due to exposure to coronary risk factors for the development of arteriosclerosis such as smoking, hypertension, diabetes, and dyslipidemia ${ }^{17}$. On the other hand, it has been revealed that vascular endothelial glycocalyx is dramatically improved due to lifestyle changes such as smoking cessation $^{18}$. In addition, vascular endothelial glycocalyx damage is also caused by a decrease in shear stress, and it is known that vascular endothelial glycocalyx damage is responsible for vulnerable plaque formation in the area of arterial bifurcations and strong flexion. In vivo and in vitro models of decreased shear stress caused vascular endothelial glycocalyx degradation, and plaque formation was strongly induced at the same site ${ }^{19}$.

\section{Molecular biological mechanism of vascular endothelial glycocalyx disorder in arteriosclerosis}

In arteriosclerosis model animals and patients with atherosclerotic diseases, vascular endothelial glycocalyx damage is induced and elevated blood levels of syndecan-1 and hyaluronan were observed. Increasing glycocalyx damage results in further thinning of the vascular endothelial glycocalyx layer. It is known that the reduction of shear stress also causes thinning of vascular endothelial glycocalyx through the inactivation of AMP-activated protein kinase (AMPK) and the activation of hyaluronidase 2 which induces shedding of hyaluronan and exfoliates vascular endothelial glycocalyx ${ }^{19}$. Syndecan-1 is known as a molecule that regulates shear stress-related signals. In syndecan-1-deficient mice, signals that induce inflammation and arteriosclerosis are activated, and vascular permeability is enhanced, and leukocyte cell adhesion on vascular endothelial cells is activated ${ }^{20}$. On the other hand, it has been 
confirmed that these signals are suppressed by the re-expression of syndecan- 1 and the progression of arteriosclerosis is suppressed. It is deeply involved in pathological conditions such as vascular hyperpermeability associated with acute inflammation and is used in many clinical studies as a major biomarker that reflects vascular endothelial glycocalyx damage.

Factors that directly damage vascular endothelial glycocalyx include reactive oxygen and nitrogen species (RNOS), uric acid ${ }^{21}$, oxidized $\mathrm{LDL}^{3}$, matrix metalloproteinases (MMPs), heparanase, sialidase, and these stimuli reduce vascular endothelial function. Administration of 4-methylumbelliferone (4$\mathrm{MU})$, a hyaluronan synthesis inhibitor, to high-fat diet-fed ApoE knockout mice showed impaired vascular endothelial glycocalyx from the early stage, increased blood pressure, and acetylcholinedependent vasorelaxation was decreased, and accelerating formation of aortic plaque with increased macrophage accumulation was clearly observed ${ }^{22}$.

On the other hand, antithrombin, TNF- $\alpha$ inhibitor ${ }^{23}$, allopurinol ${ }^{21}$, sphingosine-1 phosphate (S1P) 24, MMP inhibitors, hyaluronic acid, and steroids are reported to protect vascular endothelial glycocalyx. Since these substances are expected to exert anti-inflammatory and anti-oxidative actions, suppressive effects of both inflammation and excessive oxidative stress are expected to affect not only vascular endothelial cells but also vascular endothelial glycocalyx. It is also possible that these substances may act protectively against vascular endothelial glycocalyx.

As a morphological study, there is a report of a high-fat diet-induced arteriosclerosis model using ApoE knockout mice ${ }^{25}$. The vascular endothelial glycocalyx layer covering the plaque site was significantly thinned in the arteriosclerosis-induced mouse model, but even in the same individual, the vascular endothelial glycocalyx at the plaque-free site was not thinned. However, its thickness was similar to that of the vascular endothelial glycocalyx in normal high-fat diet-fed control mice ${ }^{25}$. Furthermore, vascular endothelial cell apoptosis was detected at a higher rate in plaque-forming sites than in nonplaque-forming sites, and shedding of the glycocalyx, promotion of plaque formation, and high expression of MMP-9 were simultaneously detected in the same sites. The vascular endothelial glycocalyx may contribute to plaque stabilization as well as maintaining the good vascular endothelial function, and the disorder is likely to play an important role in triggering plaque rupture in acute coronary syndrome.

Hyaluronic acid, which is one of the important components of vascular endothelial glycocalyx, and its binding protein, CD44, are strongly expressed in atherosclerotic lesions. The hyaluronic acid/CD44 signal activates vascular smooth muscle migration and proliferation which promotes plaque formation and increased intercellular matrix synthesis ${ }^{17}$. Further, as a mechanism of hyaluronic acid inducing atherosclerosis, hyaluronic acid activates Toll-like receptors (TLRs) to enhance the accumulation of macrophages in atherosclerotic lesions and promote Th1 cell polarization, thereby promoting macrophage immunity to promote an inflammatory response through these cells ${ }^{26}$. On the other hand, hyaluronic acid existing abundantly in the vascular endothelial glycocalyx layer exerts to suppress adhesion of leukocytes and platelets to the vascular endothelial cells. hyaluronic acid controls an inflammatory reaction in the extracellular matrix. Hyaluronic acid is known to exhibit antithrombotic and anti-inflammatory properties and is expected to suppress atherosclerosis progression by targeting the hyaluronan synthase and its receptors 27,28 .

Biglycan, a member of the class I family of small leucine-rich proteoglycans, is abundantly existing in the vascular endothelial glycocalyx layer and atherosclerotic plaque. Circulating biglycan is increased in patients with acute coronary syndrome, and also detected in the atherosclerotic plaque in ApoE knockout mice. In ApoE/biglycan double knockout mice, thrombin, platelet, and adhesion molecules were activated, as well as an increase in inflammatory cytokines and macrophage accumulation in the aortic wall ${ }^{29}$. Therefore, the loss of biglycan is considered to be closely involved in the atherosclerosis progression and plaque formation especially in the thinning layer of vascular endothelial glycocalyx.

\section{Vascular endothelial function and glycocalyx}

At the very early stage of atherosclerosis, vascular endothelial function is reduced by exposure to coronary risk factors such as hypercholesterolemia, hyperglycemia, hypertension, obesity, excessive oxidative stress, and chronic inflammation. Vascular endothelial dysfunction is observed not only in patients with early-stage of atherosclerosis but also in patients with heart failure and hypertension. 
Therefore, vascular endothelial function attracts attention as an independent prognostic factor for cardiovascular disease.

Before vascular endothelial dysfunction, vascular endothelial glycocalyx damage accompanied by a decrease in endothelium-dependent vasodilation reaction and increased vascular permeability occurs. Therefore, the vascular endothelial glycocalyx is considered a major determinant of vascular endothelial function and can be measured by the perfused boundary region (PBR) of sublingual microcirculation.

In an atherosclerotic model using ApoE/LDL receptor-deficient mice, an increased level of biomarkers associated with various vascular endothelial dysfunction was observed as a pre-stage of atherosclerotic plaque ${ }^{30}$. Specifically, circulating levels of vascular endothelial-origin biomarkers are increased like as fragmented vascular endothelial glycocalyx syndecan-1 and endothelial-cell specific molecule-1 (ESM-1), soluble vascular adhesion molecule-1 (sVCAM-1), coagulation/fibrinolysis system factor von-Willebrand-Factor (vWF), tissue-type plasminogen activator (t-PA), plasminogen activator inhibitor-1 (PAI-1), vascular hyperpermeability-related molecule of soluble fms-like tyrosine kinase-1 (sFlt-1) and angiopoietin-2. Decreased endothelium-dependent vasodilation, increased vascular endothelial permeability, and decreased NO production are already observed from the very early onset of arteriosclerosis (4 weeks after birth). In the examination of vascular endothelial function by FMD (flow-mediated dilation) using magnetic resonance imaging (MRI) and vascular endothelial glycocalyx using atomic force microscope (AFM), after the appearance of microscopic abnormalities in vascular endothelial function, vascular endothelial dysfunction, thinning of the glycocalyx layer and detachment of the vascular endothelial glycocalyx were observed. This multistep vascular endothelial damage contributes to atherosclerotic plaque formation, and a more detailed examination about the vascular endothelial glycocalyx-related mechanism is underway.

\section{Atherosclerotic risk factors and vascular endothelial glycocalyx}

Cardiovascular diseases are mainly based on atherosclerosis progression induced coronary risk factors, like smoking, hypertension, dyslipidemia, diabetes, and obesity (Fig. 4). These risk factors induce endothelial dysfunction, oxidative stress, coagulation cascade activation, inflammation, and accumulation of lifestyle diseases to stimulate further atherosclerosis progression. To improve these risk factors, lifestyle change is known to be one of the main approaches for cardiovascular disease prevention. The main objective of the total risk management for atherosclerosis patients to prevent cardiovascular disease progression is to reduce cardiovascular events and prevent chronic heart failure, and also improve their quality of life.

Figure 4. Risk factors for atherosclerosis progression and cardiovascular disease.Cardiovascular diseases are mainly caused by atherosclerosis progression induced coronary risk factors, like smoking, hypertension, dyslipidemia, diabetes, and obesity. These risk factors induce endothelial dysfunction, oxidative stress, coagulation cascade activation, inflammation and such a kind of morbidity stimulate atherosclerosis progression. CKD, chronic kidney disease.

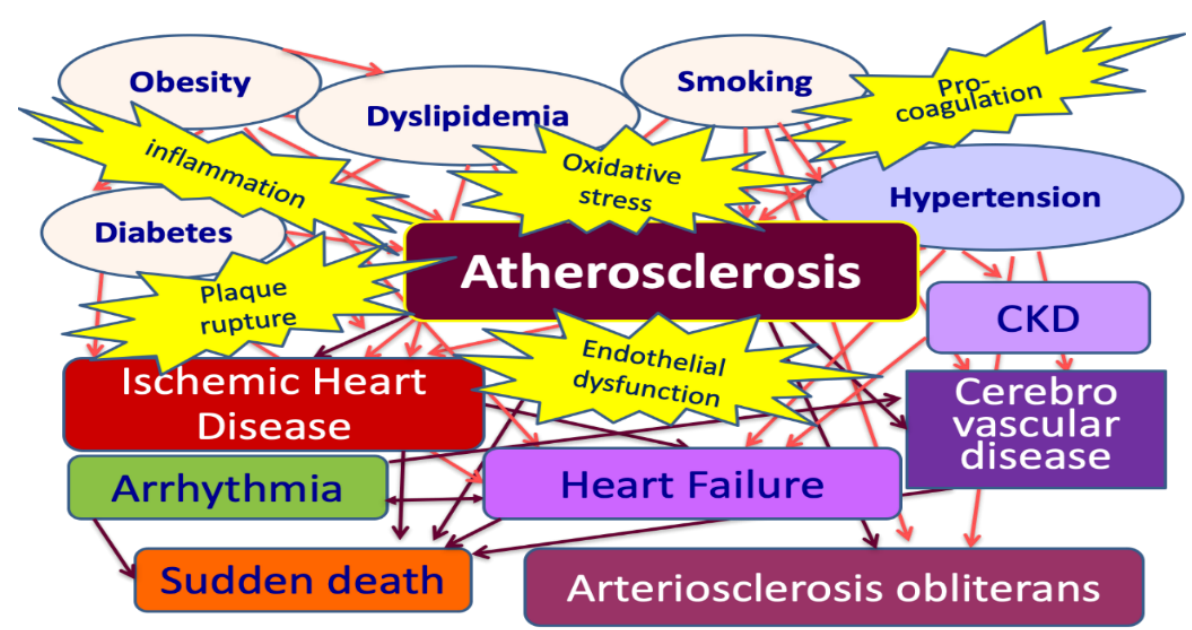


This section presents recent important findings regarding the relationship between each coronary risk factor and vascular endothelial glycocalyx are shown in this section.

\section{Diabetes}

A vascular endothelial glycocalyx, hyaluronan, is impaired in a hyperglycemic mouse model resulting in inadequate increased vascular permeability ${ }^{31}$. In a hyperglycemic model using human microvascular arterial endothelial cells (HMAECs), hyperglycemia activates TLR-2/4 signals, induces inflammation, leukocyte adhesion to HMAECs, reduces heparan sulfate expression, and increases the release of hyaluronic acid ${ }^{32}$. These signals increased the generation of excessive ROS, and contrarily antioxidants reduced TLR-2/4 gene expression and inflammatory biomarkers located its downstream. In retinal blood vessels of diabetic model rats, vascular endothelial glycocalyx of the retina was significantly thinned compared to control rats ${ }^{33}$.

In studies using therapeutic drugs for diabetes, its effectiveness has been reported in several animal models and experiments using cultured human cells. In a diabetic mice model, metformin administration for 2 weeks improved the systemic vascular endothelial glycocalyx barrier function even if in a situation where blood glucose was not affected ${ }^{34}$. Sodium glucose transporter (SGLT)-2 inhibitors are described as having a cardio/renal protective effect in recent years, it has also been reported that the incidence of cardiovascular death and exacerbation of heart failure was reduced. Empagliflozin, a SGLT-2 inhibitor, improves not only glycemic control but also reduces cardiovascular death and hospitalization for heart failure. In cultured human abdominal aortic vascular endothelial cells, empagliflozin has a protective effect on vascular endothelial glycocalyx ${ }^{35}$. The protective effect of SGLT2 inhibitors on vascular endothelial glycocalyx is considered to be one of the important mechanisms of these drugs for cardiovascular disease prevention.

\section{Dyslipidemia}

Oxidized LDL (ox-LDL) is considered to be a strong inducer of atherosclerosis progression, and its direct mechanism is to hurt vascular endothelial glycocaly $x^{3}$. It has been reported in animal studies that approximately $60 \%$ of the vascular endothelial glycocalyx is released as the shedding glycocalyx from the surface of vascular endothelial cells 25 minutes after the administration of ox-LDL, and at the same time, activation of platelet adhesion is observed on the vascular wall ${ }^{3}$. A clinical study comparing estimated whole-body vascular endothelial glycocalyx volume showed a significant decrease in patients with familial hypercholesterolemia compared to healthy subjects ${ }^{13}$. Statin therapy partially improved the volume of vascular endothelial glycocalyx, but it was significantly lower than healthy subjects.

\section{Hypertension}

Hypertension, like diabetes and dyslipidemia, causes damage to vascular endothelial glycocalyx. In the retina and choroid of hypertensive rats, vascular endothelial glycocalyx was significantly degraded and exfoliated from the blood vessels, and leukocytes and platelet adhesion to vascular endothelium were accelerated at sites where vascular endothelial glycocalyx was thinned ${ }^{33}$. In cultured vascular smooth muscle cells, glycocalyx covering the cell membrane was damaged by high-salt loading within 2-4 days, but vascular smooth muscle remodeling and cell hypertrophy were observed 6-16 days after continuous high-salt loading ${ }^{36}$. Since proliferation and hypertrophy of vascular smooth muscle are deeply involved in the progression of hypertension and arteriosclerosis caused thereby, salt reduction instruction may be effective in patients with hypertension targeting vascular endothelial glycocalyx.

Although it is known that excessive salt intake causes salt-sensitive hypertension, negatively charged vascular endothelial glycocalyx neutralizes cell surface charges by retaining sodium in the plasma in the glycocalyx layer. It is also reported that vascular endothelial glycocalyx is a regulator of salt sensitivity ${ }^{37}$. It is well known that the instruction of salt reduction as a treatment or prevention of hypertension is well known, too much salt induces myocardial cell hypertrophy even under conditions where blood pressure does not rise, and the secretion of aldosterone from the adrenal gland resulting hypertension and causing hypertrophy and fibrosis of the heart and blood vessels. Furthermore, it has 
been clarified that excessive salt intake increases urinary excretion of potassium and calcium, which may cause renal stones and osteoporosis, but potassium deficiency also increases blood pressure. Although further studies are awaited, hypernatremia-induced vascular endothelial glycocalyx damage and the activation of vascular smooth muscle remodeling should be considered to clarify the mechanism of hypertension development and arteriosclerosis progression.

\section{Chronic kidney disease (CKD)}

The involvement of albuminuria in the early stage of diabetic nephropathy has been pointed out because the clearance of negatively charged albumin is increased due to vascular endothelial glycocalyx damage ${ }^{38}$. It has been reported that glomerular vascular endothelial glycocalyx damage is caused not only by hyperglycemia and diabetic nephropathy but also by administration of adriamycin ${ }^{39}$. Among the gestational diabetes mellitus, the group in which vascular endothelial glycocalyx was released more in the blood had a higher rate of developing diabetes mellitus in the future ${ }^{40}$. From these findings, it is expected that the prognosis of the onset and exacerbation of renal damage in various diseases can be predicted by measuring the blood level of fragmented and released vascular endothelial glycocalyx.

Syndecan-1 is produced not only by vascular endothelial cells but also by glomerular epithelial cells (podocytes), and it is associated with impaired barrier function in glomerular filtration. In patients with stage 3-5 CKD, increasing levels of circulating syndecan-1 and hyaluronan were associated with decreased vascular endothelial function, and increasing levels of biomarkers related to vascular endothelial damage (sFlt-1, sVCAM-1, vWF, angiopoietin-2). Furthermore, thinning of vascular endothelial glycocalyx and increasing levels of syndecan- 1 were observed in a rat 5/6 nephrectomized CKD model ${ }^{41}$.

In patients with glomerular disease, vascular endothelial dysfunction is observed in early-stage, arteriosclerosis is likely to progress more, and cardiovascular death increases ${ }^{42}$. A cross-sectional study of patients with initial nephrotic syndrome found a significant increase in circulating syndecan-1 levels in patients with nephrotic syndrome who retained renal function ${ }^{43}$. In these patient groups, the vascular endothelial function measured by FMD was significantly lower than that in healthy subjects, and the increasing levels of syndecan-1 were closely related to the vascular endothelial dysfunction measured by FMD. Therefore, vascular endothelial glycocalyx damage accompanied by releasing its component syndecan-1 and vascular endothelial dysfunction is thought to occur at almost the same time.

In patients with advanced renal dysfunction, dialysis treatment may become necessary. However, dialysis treatment itself is a risk factor that strongly promotes arteriosclerosis. In patients undergoing dialysis therapy, the PBR of sublingual microvessels, as an important indicator for vascular endothelial glycocalyx damage, was enlarged. Moreover, circulating levels of hyaluronan and syndecan-1 were increased, and the activation of hyaluronidase was enhanced in patients with dialysis therapy ${ }^{44}$. Vascular endothelial glycocalyx damage was strongly observed in dialysis patients with high inflammatory biomarkers, and it suggests that the mechanism of atherosclerosis progression and carcinogenesis associated with decreased immune function in dialysis patients may be involved in.

\section{Cardiovascular diseases and vascular endothelial glycocalyx}

\section{Ischemic heart disease}

Circulating syndecan-1 levels were significantly higher in 141 patients with the acute coronary syndrome than that of 45 patients with chest pain syndrome without coronary artery lesions or 24 healthy subjects, and it was significantly higher especially in male patients ${ }^{45}$. The high levels of syndecan-1 in patients with the acute coronary syndrome were also significant after adjusting for sex, age, or present and past smoking 45 . In addition, circulating levels of syndecan-1 were useful to diagnose acute coronary syndrome in these patients. On the other hand, it has been reported that the blood level of syndecan- 4 was significantly higher in female patients with acute myocardial infarction ${ }^{46}$. From these results, the measurement of circulating syndecans concentration may be useful as a diagnostic biomarker to detect destabilized coronary plaque.

Compared to the rapid increase of circulating fragmented glycocalyx levels in acute diseases, circulating levels of fragmented glycocalyx was reduced in patients with chronic pathological 
conditions such as stable angina ${ }^{47}$. In such a pathological condition, thinning of the glycocalyx layer and reduction of the blood level of fragmented glycocalyx occur simultaneously due to the decreased production and accelerated degradation of the vascular endothelial glycocalyx. Therefore, the measurement of blood glycocalyx concentration is not suitable as a prognosis predictor or a severity index in chronic disease conditions.

In sublingual microvessels, vascular endothelial glycocalyx was thinned according to the severity of atherosclerotic lesions in coronary arteries, and circulating levels of hyaluronic acid in coronary arteries was significantly high in these patients ${ }^{48}$. In patients with ischemic heart disease and its highrisk patients, high PBR was extracted as a high-risk factor for arteriosclerotic cerebrovascular disease and ischemic heart disease, and PBR was associated with systolic blood pressure ${ }^{49}$. High levels of PBR are observed in patients with arteriosclerotic diseases and their high-risk groups, and the PBR measurement could be effective on disease management and screening for cardiovascular diseases. Expectations are growing for the usefulness of PBR measurement as a judgment index.

\section{Cerebrovascular disease}

Atherosclerotic cerebrovascular disease is one of the age-related diseases of the central nervous system, is related to vascular endothelial dysfunction, and is considered to be the main cause of dementia. In patients with lacunar infarction with white matter lesions, the PBR was significantly increased than those in healthy individuals or those without white matter lesions ${ }^{50}$. In a time-course analysis of endothelium-related biomarkers (chondroitin sulfate, heparin sulfate, keratan sulfate, hyaluronic acid, CD44, syndecan-1, syndecan-2, syndecan-3, syndecan-4, glypican-1, and biglycan), plasma concentrations of CD44 were significantly correlated with stroke severity in ischemic stroke patients, suggesting a relationship between CD44 and brain tissue damage and infarct size ${ }^{51}$. The measurement of PBR in arteriosclerotic diseases may be useful in predicting its severity and prognosis, but further study is necessary.

Hyaluronic acid concentrations in cerebrospinal fluid of 46 patients with cerebrovascular dementia were significantly higher compared to 45 patients with Alzheimer's disease and 26 healthy groups without dementia ${ }^{52}$. Regarding the hyaluronic acid in cerebrospinal fluid, vascular endothelial glycocalyx damage in cerebral blood vessels has been suggested that vascular endothelial glycocalyx damage in the microcirculation of various organs is closely related to the damage of various organs.

\section{On a final note}

It is said that vascular endothelial glycocalyx is a primitive barrier function, but the gel-like component consisting of macromolecules such as sugar chains and glycoproteins flexibly change their density and composition according to the state of the living body. Vascular endothelial glycocalyx encapsulates various substances, provides various molecules for maintaining homeostasis in the body, and regulates signals of vascular endothelial cells. Although the direct investigation of the in vivo function of vascular endothelial glycocalyx throughout the living body is complicated, vascular endothelial glycocalyx has great potential to explore new strategies for assessing the benefit conditions of our healthy vasculature. 


\section{References}

1. van den Berg BM, Vink H and Spaan JA. The endothelial glycocalyx protects against myocardial edema. Circ Res. 2003;92:592-4.

2. Rorije NMG, Rademaker E, Schrooten EM, Wouda RD, Homan Van Der Heide JJ, Van Den Born BH and Vogt L. High-salt intake affects sublingual microcirculation and is linked to body weight change in healthy volunteers: a randomized cross-over trial. J Hypertens. 2019;37:1254-1261.

3. Vink H, Constantinescu AA and Spaan JA. Oxidized lipoproteins degrade the endothelial surface layer : implications for platelet-endothelial cell adhesion. Circulation. 2000;101:1500-2.

4. Broekhuizen LN, Lemkes BA, Mooij HL, Meuwese MC, Verberne H, Holleman F, Schlingemann RO, Nieuwdorp M, Stroes ES and Vink H. Effect of sulodexide on endothelial glycocalyx and vascular permeability in patients with type 2 diabetes mellitus. Diabetologia. 2010;53:2646-55.

5. Groen BB, Hamer HM, Snijders T, van Kranenburg J, Frijns D, Vink H and van Loon LJ. Skeletal muscle capillary density and microvascular function are compromised with aging and type 2 diabetes. J Appl Physiol (1985). 2014;116:998-1005.

6. Ostrowski SR, Gaini S, Pedersen C and Johansson PI. Sympathoadrenal activation and endothelial damage in patients with varying degrees of acute infectious disease: an observational study. J Crit Care. 2015;30:906.

7. Ohnishi Y, Yasudo H, Suzuki Y, Furuta T, Matsuguma C, Azuma Y, Miyake A, Okada S, Ichihara K, Ohga $\mathrm{S}$ and Hasegawa S. Circulating endothelial glycocalyx components as a predictive marker of coronary artery lesions in Kawasaki disease. Int J Cardiol. 2019;292:236-240.

8. Steppan J, Hofer S, Funke B, Brenner T, Henrich M, Martin E, Weitz J, Hofmann U and Weigand MA. Sepsis and major abdominal surgery lead to flaking of the endothelial glycocalix. J Surg Res. 2011;165:136-41.

9. Rubio-Gayosso I, Platts SH and Duling BR. Reactive oxygen species mediate modification of glycocalyx during ischemia-reperfusion injury. Am J Physiol Heart Circ Physiol. 2006;290:H2247-56.

10. Chignalia AZ, Yetimakman F, Christiaans SC, Unal S, Bayrakci B, Wagener BM, Russell RT, Kerby JD, Pittet JF and Dull RO. The Glycocalyx and Trauma: A Review. Shock. 2016;45:338-48.

11. Schmidt EP, Overdier KH, Sun X, Lin L, Liu X, Yang Y, Ammons LA, Hiller TD, Suflita MA, Yu Y, Chen Y, Zhang F, Cothren Burlew C, Edelstein CL, Douglas IS and Linhardt RJ. Urinary Glycosaminoglycans Predict Outcomes in Septic Shock and Acute Respiratory Distress Syndrome. Am J Respir Crit Care Med. 2016;194:439-49.

12. Weissgerber TL, Garcia-Valencia O, Milic NM, Codsi E, Cubro H, Nath MC, White WM, Nath KA and Garovic VD. Early Onset Preeclampsia Is Associated With Glycocalyx Degradation and Reduced Microvascular Perfusion. J Am Heart Assoc. 2019;8:e10647.

13. Meuwese MC, Mooij HL, Nieuwdorp M, van Lith B, Marck R, Vink H, Kastelein JJ and Stroes ES. Partial recovery of the endothelial glycocalyx upon rosuvastatin therapy in patients with heterozygous familial hypercholesterolemia. J Lipid Res. 2009;50:148-53.

14. Wadowski PP, Hulsmann M, Schorgenhofer C, Lang IM, Wurm R, Gremmel T, Koppensteiner R, Steinlechner B, Schwameis $\mathrm{M}$ and Jilma B. Sublingual functional capillary rarefaction in chronic heart failure. Eur J Clin Invest. 2018;48.

15. Jaarsma C, Vink H, van Haare J, Bekkers S, van Rooijen BD, Backes WH, Wildberger JE, Crijns HJ, van Teeffelen J and Schalla S. Non-invasive assessment of microvascular dysfunction in patients with microvascular angina. Int J Cardiol. 2017;248:433-439.

16. Marechal X, Favory R, Joulin O, Montaigne D, Hassoun S, Decoster B, Zerimech F and Neviere R. Endothelial glycocalyx damage during endotoxemia coincides with microcirculatory dysfunction and vascular oxidative stress. Shock. 2008;29:572-6.

17. Nieuwdorp M, Meuwese MC, Vink H, Hoekstra JB, Kastelein JJ and Stroes ES. The endothelial glycocalyx: a potential barrier between health and vascular disease. Curr Opin Lipidol. 2005;16:507-11.

18. Ikonomidis I, Marinou M, Vlastos D, Kourea K, Andreadou I, Liarakos N, Triantafyllidi H, Pavlidis G, Tsougos E, Parissis J and Lekakis J. Effects of varenicline and nicotine replacement therapy on arterial elasticity, endothelial glycocalyx and oxidative stress during a 3-month smoking cessation program. Atherosclerosis. 2017;262:123-130.

19. Zhang J, Kong X, Wang Z, Gao X, Ge Z, Gu Y, Ye P, Chao Y, Zhu L, Li X and Chen S. AMP-activated protein kinase regulates glycocalyx impairment and macrophage recruitment in response to low shear stress. 
FASEB J. 2019;33:7202-7212.

20. Voyvodic PL, Min D, Liu R, Williams E, Chitalia V, Dunn AK and Baker AB. Loss of syndecan-1 induces a pro-inflammatory phenotype in endothelial cells with a dysregulated response to atheroprotective flow. J Biol Chem. 2014;289:9547-59.

21. Ko J, Kang HJ, Kim DA, Kim MJ, Ryu ES, Lee S, Ryu JH, Roncal C, Johnson RJ and Kang DH. Uric acid induced the phenotype transition of vascular endothelial cells via induction of oxidative stress and glycocalyx shedding. FASEB J. 2019:fj201901148R.

22. Nagy N, Freudenberger T, Melchior-Becker A, Rock K, Ter Braak M, Jastrow H, Kinzig M, Lucke S, Suvorava T, Kojda G, Weber AA, Sorgel F, Levkau B, Ergun S and Fischer JW. Inhibition of hyaluronan synthesis accelerates murine atherosclerosis: novel insights into the role of hyaluronan synthesis. Circulation. 2010;122:2313-22.

23. Nieuwdorp M, Meuwese MC, Mooij HL, van Lieshout MH, Hayden A, Levi M, Meijers JC, Ince C, Kastelein JJ, Vink $\mathrm{H}$ and Stroes ES. Tumor necrosis factor-alpha inhibition protects against endotoxin-induced endothelial glycocalyx perturbation. Atherosclerosis. 2009;202:296-303.

24. Mensah SA, Cheng MJ, Homayoni H, Plouffe BD, Coury AJ and Ebong EE. Regeneration of glycocalyx by heparan sulfate and sphingosine 1-phosphate restores inter-endothelial communication. PLoS One. 2017;12:e0186116.

25. Cancel LM, Ebong EE, Mensah S, Hirschberg C and Tarbell JM. Endothelial glycocalyx, apoptosis and inflammation in an atherosclerotic mouse model. Atherosclerosis. 2016;252:136-146.

26. Mullick AE, Tobias PS and Curtiss LK. Modulation of atherosclerosis in mice by Toll-like receptor 2. J Clin Invest. 2005;115:3149-56.

27. Fischer JW. Role of hyaluronan in atherosclerosis: Current knowledge and open questions. Matrix Biol. 2019;78-79:324-336.

28. Grandoch M, Bollyky PL and Fischer JW. Hyaluronan: A Master Switch Between Vascular Homeostasis and Inflammation. Circ Res. 2018;122:1341-1343.

29. Grandoch M, Kohlmorgen C, Melchior-Becker A, Feldmann K, Homann S, Muller J, Kiene LS, ZengBrouwers J, Schmitz F, Nagy N, Polzin A, Gowert NS, Elvers M, Skroblin P, Yin X, Mayr M, Schaefer L, Tannock LR and Fischer JW. Loss of Biglycan Enhances Thrombin Generation in Apolipoprotein EDeficient Mice: Implications for Inflammation and Atherosclerosis. Arterioscler Thromb Vasc Biol. 2016;36:e41-50.

30. Bar A, Targosz-Korecka M, Suraj J, Proniewski B, Jasztal A, Marczyk B, Sternak M, Przybylo M, Kurpinska A, Walczak M, Kostogrys RB, Szymonski M and Chlopicki S. Degradation of Glycocalyx and Multiple Manifestations of Endothelial Dysfunction Coincide in the Early Phase of Endothelial Dysfunction Before Atherosclerotic Plaque Development in Apolipoprotein E/Low-Density Lipoprotein Receptor-Deficient Mice. J Am Heart Assoc. 2019;8:e011171.

31. Zuurbier CJ, Demirci C, Koeman A, Vink H and Ince C. Short-term hyperglycemia increases endothelial glycocalyx permeability and acutely decreases lineal density of capillaries with flowing red blood cells. J Appl Physiol (1985). 2005;99:1471-6.

32. Pahwa R, Nallasamy $\mathrm{P}$ and Jialal I. Toll-like receptors 2 and 4 mediate hyperglycemia induced macrovascular aortic endothelial cell inflammation and perturbation of the endothelial glycocalyx. J Diabetes Complications. 2016;30:563-72.

33. Kumase F, Morizane Y, Mohri S, Takasu I, Ohtsuka A and Ohtsuki H. Glycocalyx degradation in retinal and choroidal capillary endothelium in rats with diabetes and hypertension. Acta Med Okayama. 2010;64:277-83.

34. Eskens BJ, Zuurbier CJ, van Haare J, Vink H and van Teeffelen JW. Effects of two weeks of metformin treatment on whole-body glycocalyx barrier properties in db/db mice. Cardiovasc Diabetol. 2013;12:175.

35. Cooper S, Teoh H, Campeau MA, Verma S and Leask RL. Empagliflozin restores the integrity of the endothelial glycocalyx in vitro. Mol Cell Biochem. 2019;459:121-130.

36. Bkaily G, Simon Y, Menkovic I, Bkaily C and Jacques D. High salt-induced hypertrophy of human vascular smooth muscle cells associated with a decrease in glycocalyx. J Mol Cell Cardiol. 2018;125:1-5.

37. Oberleithner $\mathrm{H}$ and Wilhelmi M. Vascular glycocalyx sodium store - determinant of salt sensitivity? Blood Purif. 2015;39:7-10.

38. Jeansson M, Granqvist AB, Nystrom JS and Haraldsson B. Functional and molecular alterations of the glomerular barrier in long-term diabetes in mice. Diabetologia. 2006;49:2200-9. 
39. Jeansson M, Bjorck K, Tenstad $\mathrm{O}$ and Haraldsson B. Adriamycin alters glomerular endothelium to induce proteinuria. J Am Soc Nephrol. 2009;20:114-22.

40. Long DS, Hou W, Taylor RS and McCowan LM. Serum levels of endothelial glycocalyx constituents in women at 20 weeks' gestation who later develop gestational diabetes mellitus compared to matched controls: a pilot study. BMJ Open. 2016;6:e011244.

41. Padberg JS, Wiesinger A, di Marco GS, Reuter S, Grabner A, Kentrup D, Lukasz A, Oberleithner H, Pavenstadt H, Brand M and Kumpers P. Damage of the endothelial glycocalyx in chronic kidney disease. Atherosclerosis. 2014;234:335-43.

42. Watts GF, Herrmann S, Dogra GK, Playford DA, Best JD, Thomas MA and Irish A. Vascular function of the peripheral circulation in patients with nephrosis. Kidney Int. 2001;60:182-9.

43. Salmito FT, de Oliveira Neves FM, Meneses GC, de Almeida Leitao R, Martins AM and Liborio AB. Glycocalyx injury in adults with nephrotic syndrome: Association with endothelial function. Clin Chim Acta. 2015;447:55-8.

44. Vlahu CA, Lemkes BA, Struijk DG, Koopman MG, Krediet RT and Vink H. Damage of the endothelial glycocalyx in dialysis patients. J Am Soc Nephrol. 2012;23:1900-8.

45. Miranda $\mathrm{CH}$, de Carvalho Borges M, Schmidt A, Marin-Neto JA and Pazin-Filho A. Evaluation of the endothelial glycocalyx damage in patients with acute coronary syndrome. Atherosclerosis. 2016;247:184-8.

46. Solbu MD, Kolset SO, Jenssen TG, Wilsgaard T, Lochen ML, Mathiesen EB, Melsom T, Eriksen BO and Reine TM. Gender differences in the association of syndecan- 4 with myocardial infarction: The populationbased Tromso Study. Atherosclerosis. 2018;278:166-173.

47. Nemoto T, Minami Y, Yamaoka-Tojo M, Kato A, Katsura A, Sato T, Muramatsu Y, Kakizaki R, Fujiyoshi K, Hashimoto T, Meguro K, Shimohama T and Ako J. Endothelial glycocalyx and severity and vulnerability of coronary plaque in patients with coronary artery disease. Atherosclerosis. 2020;302:1-7.

48. Xue XJ, Jiang Y, Chen L and Chen SL. Relationship between the endothelial glycocalyx and the extent of coronary atherosclerosis. Microcirculation. 2018;25:e12504.

49. Gorshkov AY, Klimushina MV, Boytsov SA, Kots AY and Gumanova NG. Increase in perfused boundary region of endothelial glycocalyx is associated with higher prevalence of ischemic heart disease and lesions of microcirculation and vascular wall. Microcirculation. 2018;25:e12454.

50. Martens RJ, Vink H, van Oostenbrugge RJ and Staals J. Sublingual microvascular glycocalyx dimensions in lacunar stroke patients. Cerebrovasc Dis. 2013;35:451-4.

51. DellaValle B, Hasseldam H, Johansen FF, Iversen HK, Rungby J and Hempel C. Multiple Soluble Components of the Glycocalyx Are Increased in Patient Plasma After Ischemic Stroke. Stroke. 2019;50:29482951.

52. Nagga K, Hansson O, van Westen D, Minthon L and Wennstrom M. Increased levels of hyaluronic acid in cerebrospinal fluid in patients with vascular dementia. J Alzheimers Dis. 2014;42:1435-41. 